\title{
Fast crystal growth in glass-forming liquids
}

\author{
J. Orava, ${ }^{\mathrm{a}, \mathrm{b},{ }^{*}}$ A. L. Greer ${ }^{\mathrm{a}, \mathrm{b}, *}$
}

${ }^{\mathrm{a}}$ University of Cambridge, Department of Materials Science \& Metallurgy 27 Charles Babbage Road, Cambridge CB3 OFS, UK

${ }^{\mathrm{b}}$ WPI Advanced Institute for Materials Research, Tohoku University, 2-1-1 Katahira, Aobaku, Sendai 980-8577, Japan

Corresponding authors: jo316@cam.ac.uk (JO); alg13@cam.ac.uk (ALG)

\begin{abstract}
In liquids of high glass-forming ability, in which crystal growth rates are low, the rates can be measured over the full range of supercooling from the liquidus temperature down to the glass transition. For systems of low glass-forming ability, growth rates are readily measured at small supercooling and at very large supercooling around the glass-transition temperature, but it is difficult to acquire data over the full range of intermediate supercooling, especially at the maximum in growth rate. Data at intermediate supercoolings are however of considerable interest for understanding glass formation in such systems as pure metals and chalcogenides for phase-change data storage. We will review the methods emerging for making such measurements, and will note that the fragility of the liquid (including possible crossover from 'fragile' to 'strong' liquid behaviour on cooling) is an important part of understanding fast crystal growth. We also note that there are deficiencies in existing theories of fast crystal growth.
\end{abstract}

Keywords: Crystal growth, Fragility, Glasses, Viscosity,

MSC Classification Codes: 82B26, 82D15

\section{Introduction}

Glass formation on cooling a liquid involves avoidance of crystallization, and analysing glass-forming ability (GFA) requires the kinetics of both crystal nucleation and growth to be taken into account. In this review, however, we focus only on the rates of crystal growth $U$. Furthermore, to avoid complications from solute partitioning, we restrict ourselves to systems that crystallize congruently (i.e. the liquid/glass and the single-phase crystal forming from it have identical compositions). High GFA is associated with sluggish crystallization, but there is much current interest in marginal glass-forming systems in which $U$ can be high. Most 
liquids, including it now seems pure-metal melts [1,2], can form glasses if cooled sufficiently rapidly, at $>10^{10} \mathrm{~K} \mathrm{~s}^{-1}$. For glass-forming systems, it is well accepted schematically, but rarely measured quantitatively, that $U$ must exhibit a maximum in the temperature range between the melting point $T_{\mathrm{m}}$ (equivalent to the liquidus temperature when freezing is congruent) and the glass-transition temperature $T_{\mathrm{g}}$. For classic glass-formers, such as silica and the oxide glasses, the values of $U$ are low and can be measured (often by direct observation of the crystals) over the full range of supercooling from $T_{\mathrm{m}}$ down to $T_{\mathrm{g}}$. Even for poor glass-formers, such as most metallic systems, large supercoolings can be achieved in levitated droplets, and there are now many measurements of rapid crystal growth [3]. But so far only one study (on $\mathrm{Cu}_{50} \mathrm{Zr}_{50}$ [4]) has reached supercoolings large enough to permit measurement of the maximum $U_{\max }$ in crystal growth rate. There are many measurements of $U$ in the glass (Sect. 5) and supercooled-liquid states near $T_{\mathrm{g}}$, but for poor glass-formers it is difficult to acquire data over the intermediate supercoolings relevant for $U_{\max }$. Yet such data are not only of fundamental interest, for example in setting the ultimate limits to glass stability, but also of technological importance for the operation of phase-change memory (where the materials of current interest are chalcogenides).

Our focus is on the measurement and analysis of fast crystal growth, especially in the temperature range of $U_{\max }$. We note that the mode of crystallization in this regime may well be different from that at small supercooling; in particular the high driving force permits fast congruent freezing to a metastable phase, favoured by the lack of the inhibiting factor of solute partitioning. Property changes in the liquid as a function of temperature are not of concern in conventional solidification studies, where the liquid supercooling is always small. But such changes are important over the wide range of supercooling relevant for glassforming systems, and provide further points of interest. We will review the methods emerging for making measurements of $U$, emphasizing those relevant for fast growth in systems of low GFA, and will note that the fragility of the liquid (including possible crossover from 'fragile' to 'strong' liquid behaviour on cooling) can be an important part of understanding crystal growth rates.

\section{Liquid and glassy states}

It is familiar that different glassy states are formed by cooling the liquid at different rates. The range of states that can be achieved has been explored particularly intensively for metallic glass-forming systems [5]. We take such a system to show the range of enthalpies of interest: Fig. 1 is schematic, but based on calorimetric data for the system $\mathrm{Zr}_{52.5} \mathrm{Cu}_{17.9} \mathrm{Ni}_{14.6} \mathrm{Al}_{10.0} \mathrm{Ti}_{5.0}$ (composition in at.\%) forming the bulk metallic glass Vitreloy 105 [6]. The $T_{\mathrm{g}}$ indicated in the figure is for the standard cooling rate of $0.33 \mathrm{~K} \mathrm{~s}^{-1}$, and corresponds to the conventionally adopted value of viscosity $\eta=10^{12} \mathrm{~Pa}$ s. The heat of 
crystallization $\Delta H_{\mathrm{x}}$ of a relaxed glass, measured using conventional differential scanning calorimetry (DSC) at a typical heating rate of $\sim 10^{-1} \mathrm{~K} \mathrm{~s}^{-1}$, is $\sim 0.4 \Delta H_{\mathrm{m}}$ (where $\Delta H_{\mathrm{m}}$ is the latent heat of melting). The large difference between $\Delta H_{\mathrm{x}}$ and $\Delta H_{\mathrm{m}}$ indicates that there is considerable ordering in the liquid as it is supercooled. States of different order are retained in the glass by cooling at different rates. Figure 1 shows rates from $10^{13} \mathrm{~K} \mathrm{~s}^{-1}$ (possibly achieved, or exceeded, experimentally in pure-metal nanobridges [1]) down to $10^{-14} \mathrm{~K} \mathrm{~s}^{-1}$ (the effective cooling rate that would correspond to ultrastable states obtainable by deposition with a glass-transition temperature at about $85 \%$ of the conventional value). For such a wide range of cooling rates, the range of enthalpy is very wide, up to $\sim 0.9 \Delta H_{\mathrm{m}}$ [5]. In principle, analysis of crystal growth rates in glass-forming liquids should consider such a wide range of states. For any glassy state, the details of the transition to the liquid state on heating depend on the heating rate (inset in Fig. 1). On heating, usually the glass undergoes relaxation and goes through $T_{\mathrm{g}}$ before crystallization; the nature of the crystallization must then be unaffected by the prior glassy state. For glassy states of very high enthalpy (rejuvenated states), however, crystallization may occur below $T_{\mathrm{g}}$ [2].

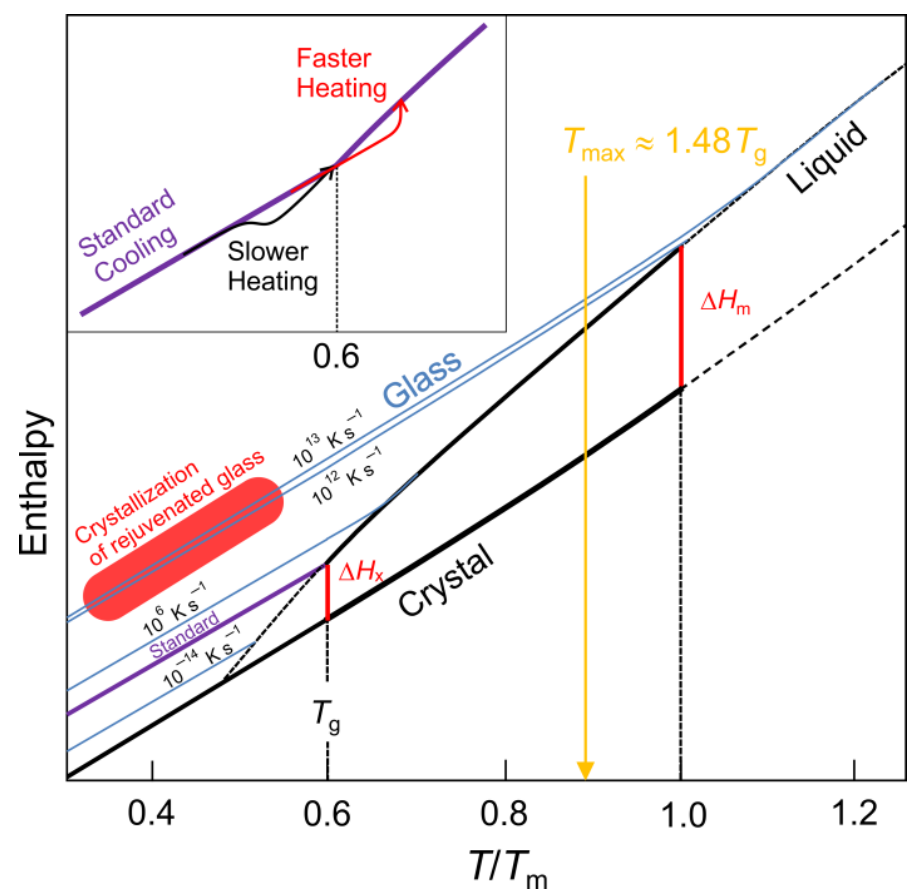

Fig. 1. Relative enthalpy as a function of reduced temperature for a bulk-metallic-glass forming system, showing representative scaling between the specific heats $(\mathrm{d} H / \mathrm{d} T)$ and the heat of melting $\Delta H_{\mathrm{m}} . T_{\max }$ is the temperature at which the crystal growth rate reaches its maximum $U_{\max }$. The inset shows the relaxation behaviour near $T_{\mathrm{g}}$ under differing heating rates. At a given temperature, the enthalpy range in potential glassy states is remarkably wide, up to $\sim 0.9 \Delta H_{\mathrm{m}}$. (Adapted from Ref. [5])

Our interest is mostly in the maximum growth rate $U_{\max }$, which occurs at a temperature $T_{\max }$ between $T_{\mathrm{g}}$ and $T_{\mathrm{m}}$. It has been shown for a wide range of glass-forming systems (data 
for some of these are included in Fig. 2) that there is a good correlation between $T_{\max }$ and $T_{\mathrm{g}}$, with $T_{\max }=(1.48 \pm 0.15) T_{\mathrm{g}}$ [7]. On that basis, $T_{\max }$ is indicated in Fig. 1 , and we see that at this temperature, far above the standard $T_{\mathrm{g}}$, only a small range of enthalpy would be relevant for the maximum in crystal growth rate. The value of $U_{\max }$ would be affected only by quenching at the very highest rates not relevant for our current interest (though relevant for moleculardynamics simulations). While there can be non-equilibrium effects in supercooled liquids (e.g. analysed in terms of retarded viscosity [8]), we conclude that in the current work, with its focus near $T_{\max }$, we can take the properties of the liquid to be characteristic of the actual temperature (i.e. independent of thermal history, cooling or heating rate).

\section{Influence of reduced glass-transition temperature and fragility}

Turnbull [9] noted that the reduced glass-transition temperature, $T_{\mathrm{rg}}\left(=T_{\mathrm{g}} / T_{\mathrm{m}}\right)$ is an important parameter governing GFA. He considered the rate, $I$, of homogeneous nucleation of crystals in the liquid, and showed that nucleation is effectively suppressed when $T_{\mathrm{rg}}>0.67$. As $T_{\text {rg }}$ increases, the temperature at which $I$ is maximum increases, and the maximum value of $I$ decreases markedly. He also noted that the correlation of $I(T)$ with $T_{\mathrm{rg}}$ would be affected by different liquids having different temperature dependences of viscosity $\eta(T)$. In the current literature this is discussed in terms of the kinetic fragility of the liquid, defined as $m=\left\lfloor\mathrm{d}\left(\log _{10} \eta\right) / \mathrm{d}\left(T_{\mathrm{g}} / T\right)\right\rfloor_{T=T_{\mathrm{g}}}[10]$. Senkov [11] has suggested that GFA can be correlated with a combination of the two independent parameters $T_{\mathrm{rg}}$, and $m$, and he defined a dimensionless parameter, $F=2\left[(m / 16)\left(1 / T_{\mathrm{rg}}-1\right)+2\right]^{-1}$, with $F$ varying from $\sim 0.1$ for very fragile liquids to $\sim 0.8$ for strong liquid $\mathrm{SiO}_{2}$.

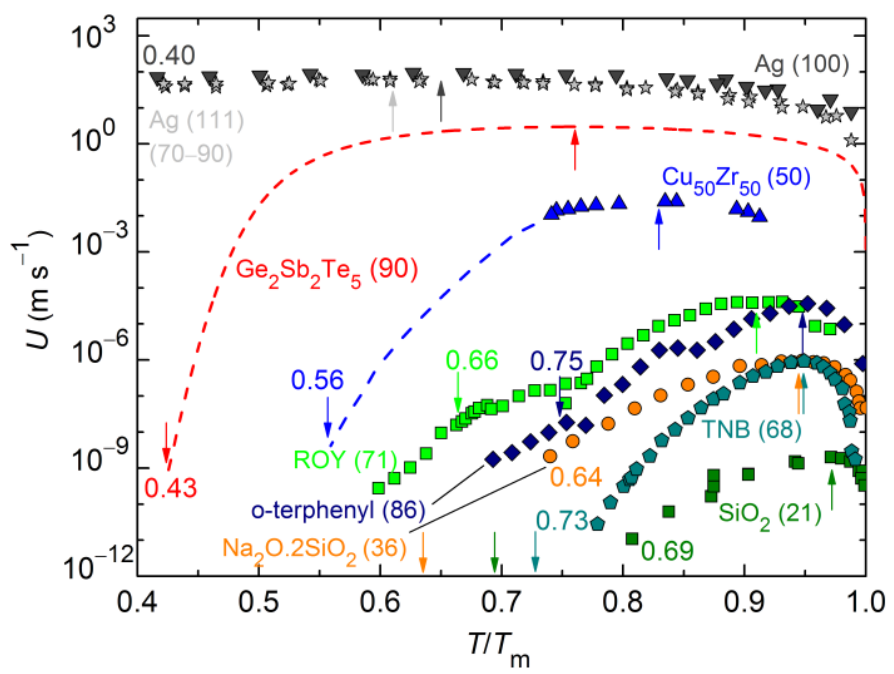

Fig. 2. Survey of crystal growth rates in variety of glass-forming liquids showing congruent freezing. The upward arrows indicate the maximum in the crystal growth rate; the downward arrows mark the reduced glasstransition temperature $T_{\mathrm{rg}}$, and the values are given. The numbers in parentheses show values of the liquid fragility $m$. Modified from Ref. [7]. 
Our previous work [7] examined how $T_{\mathrm{rg}}$ and $m$ might affect the temperature dependence of crystal growth rate $U(T)$ in supercooled liquids. The compilation of $U(T)$ data for diverse glass-forming systems (Fig. 2), remarkably resembles Turnbull's plot of $I(T)$ for different values of $T_{\mathrm{rg}}$. Although there are relevant studies of polymeric crystallization [12], we exclude polymeric systems from the comparison in Fig. 2; as noted in earlier work [7,13], in such systems, factors such as chain length disrupt the usual correlation between crystal growth rate and viscosity. In Fig. 2, the values of the maximum growth rate $U_{\max }$ range over nearly 11 orders of magnitude, and, for a given span of $T_{\mathrm{rg}}$ values, this range is essentially the same as that of the maximum in $I$. Thus growth rates are also important in understanding GFA. For a high-GFA system, the value of $U_{\max }$ is low and occurs at high value of $T_{\max } / T_{\mathrm{m}}$ (e.g. 0.97 for $\mathrm{SiO}_{2}$ ). For a low-GFA system, $U(T)$ shows a much broader peak, $U_{\max }$ is high, and occurs at a low value of $T_{\max } / T_{\mathrm{m}}$ (e.g. 0.61 for pure silver, obtained from moleculardynamics simulations).

In Fig. 2, values of $T_{\mathrm{rg}}$ and $m$ are given for each set of data. The values of $U_{\max }$ do not show good correlations with a single parameter $T_{\mathrm{rg}}$ or $m$. But this earlier work [7] established, adopting a strategy similar to that of Senkov [11], that $U_{\max }$ correlates well with a linear combination of $T_{\mathrm{rg}}$ and $m$ that can be regarded as a fragility-corrected $T_{\mathrm{rg}}$ :

$$
T_{\mathrm{gu}}=T_{\mathrm{rg}}-\frac{m}{\chi} .
$$

The correlation of $U_{\max }$ with $T_{\mathrm{gu}}$ over the entire range of glass-forming systems, setting $\chi=$ 505, is shown in Fig. 3. A remarkably similar fragility-corrected $T_{\text {rg }}$ appears to explain, with a similar value of $\chi=520$, the relative GFAs of Ni-Cr-Nb-P-B alloys [14]; with more data, on a total of 42 metallic-glass-forming liquids, a best fitting gives $\chi=532$ [15]. 


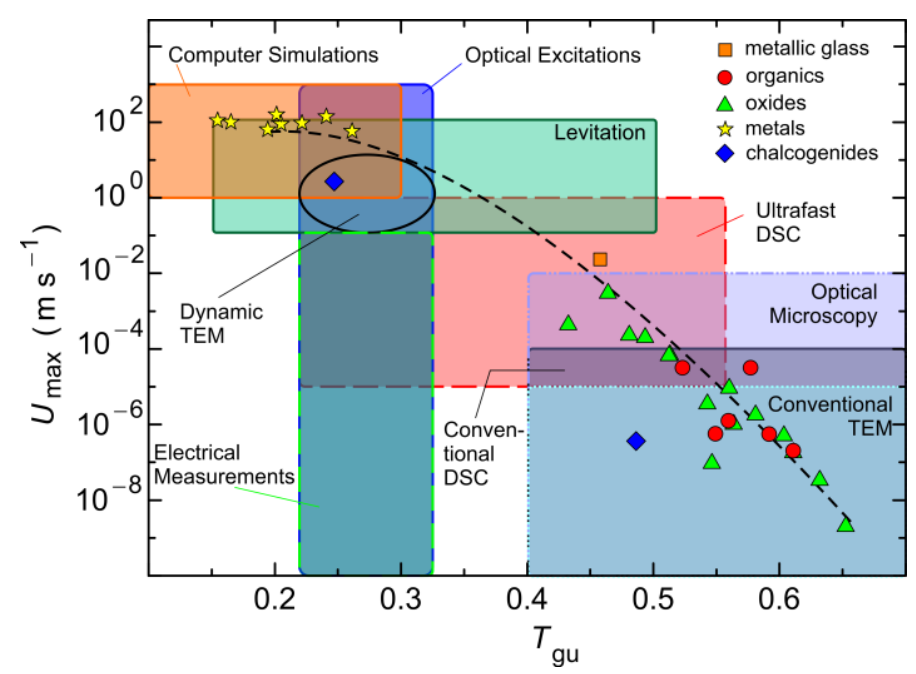

Fig. 3. Correlation between $U_{\max }$ and the fragility-compensated reduced-glass-transition temperature $T_{\mathrm{gu}}$ (equation 1). The coloured boxes show schematically typical ranges of $U_{\max }$ and $T_{\mathrm{gu}}$ that are measured by a variety of direct and indirect techniques (Tab. 1, Sect. 4). Modified from Ref. [7].

Independent of the underlying influences of $T_{\mathrm{rg}}$ and $m$, Fig. 2 suggests a general correlation of $U_{\max }$ vs $T_{\max } / T_{\mathrm{m}}$, and this is shown in Fig. 4 [7,16]. This shows scatter, some of which may be because the actual interface temperature is not well established in some experiments (methods and the limitations are treated in Sect. 4). There is also the influence of diverse growth mechanisms. Nevertheless, the correlation in Fig. 4 does provide a basis for a generalized description of crystal growth rate in glass-forming liquids, as developed by Schmelzer et al. [16] who derived the following master equation:

$$
\left.U_{\max }\left(T_{\max }\right) \cong f \frac{D_{0}}{4 d_{0}}\left(\frac{\frac{q}{E_{\mathrm{D}}^{\mathrm{eff}}}}{1+\frac{q}{E_{\mathrm{D}}^{\mathrm{eff}}}}\right) \exp \left(-\frac{1}{1-\left(\frac{T_{\max }}{T_{\mathrm{m}}}\right)}\right)\right|_{T=T_{\max }},
$$

where $f \leq 1$ is the fraction of active growth sites at the interface accounting for different growth modes ( $f=1$ for a normal growth, $f \approx \Delta T / 2 \pi T_{\mathrm{m}}$ for a dislocation-mediated growth), $D_{0}$ is the pre-exponential factor of the temperature-dependent diffusion coefficient $D(T), d_{0}$ is the molecular diameter, $q$ is the latent heat of crystallization per molecule, and $E_{\mathrm{D}}^{\mathrm{eff}}$ is the thermally-compensated activation energy for diffusion. Calculations based on Eq. (2) (solid blue circles, for oxides) [16] are compared with the measured data in Figure 4; the match is encouraging. 


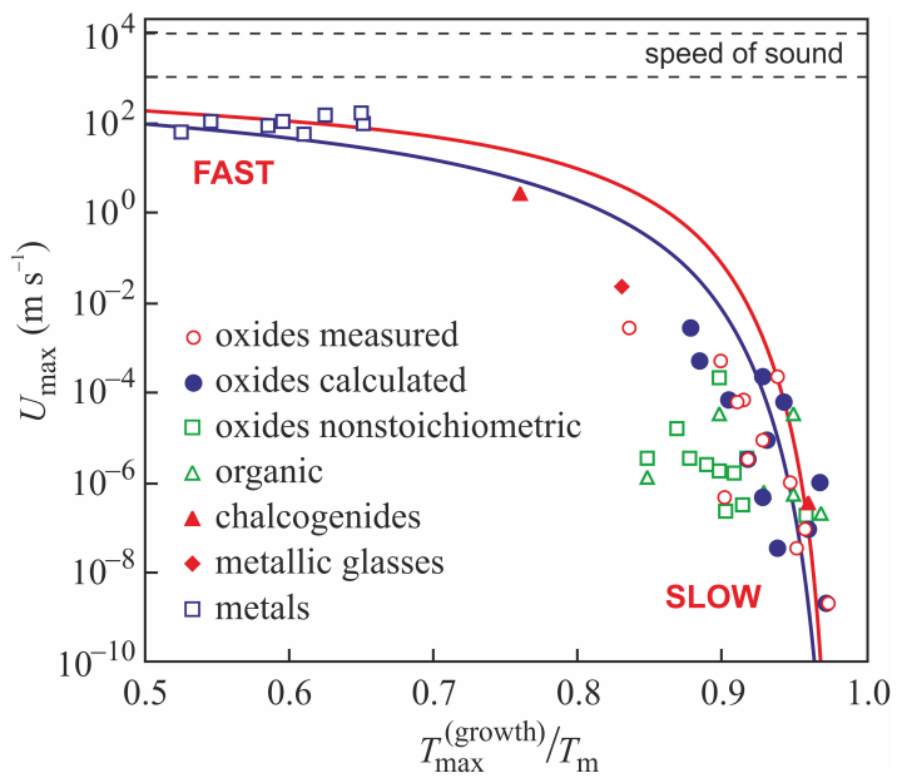

Fig. 4. Correlation between measured and calculated (Eq. 2 - solid blue circles) maximum in crystal growth rate $U_{\max }$ and homologous temperature $T_{\max } / T_{\mathrm{m}}$. The red and blue solid lines show calculations for normal and dislocation-mediated growth, respectively. Published with permission from Ref. [16].

\section{Measurement of crystal growth rates}

It is evident from Fig. 2 and Fig. 3 that the values of $U_{\max }$ span a wide range. In Fig. 3, we show the typical ranges over which different techniques may be useful for the measurement of growth rates. The techniques are both direct and indirect, as summarized in Table 1. The direct techniques each involve the observation of the advance of crystallization front. Even so, there may be uncertainties: for example, for higher values of $U$, the interface temperature may not be well known, significantly exceeding the temperature of the bulk sample. In optical microscopy, and electron microscopy, the front is directly observed; in experiments on levitated droplets, the position of the interface is inferred from the thermal front that crosses the droplet during solidification.

\section{Table 1}

A variety of experimental techniques, indirect and direct, are used to measure crystal growth rates in glassforming liquids (Fig. 3). Typical ranges of the maximum rate $U_{\max }$ and of the fragility-corrected reduced glasstransition temperature $T_{\mathrm{gu}}$ (Eq. 1) probed by the techniques at varying heating/cooling rates are summarized.

\begin{tabular}{|c|c|c|c|c|}
\hline Indirect Techniques & $\begin{array}{c}U_{\max } \\
\left(\mathrm{m} \mathrm{s}^{-1}\right)\end{array}$ & $T_{\mathrm{gu}}$ & $\begin{array}{c}\text { Heating/Cooling } \\
\text { Rate }\left(\mathrm{K} \mathrm{s}^{-\mathbf{1}}\right)\end{array}$ & Limitations \\
\hline Computer Simulations & $10^{0}-10^{3}$ & $0.1-0.3$ & $10^{11}-10^{15} / 10^{11}-10^{15}$ & $\begin{array}{c}\text { Accurate interatomic } \\
\text { potentials are needed }^{\text {a) }}[17,18]\end{array}$ \\
\hline Conventional DSC & $<10^{-4}$ & $0.4-0.7$ & $10^{-4}-10^{0} / 10^{-4}-10^{-1}$ & Arrhenius kinetics ${ }^{\text {b) }}[19,20]$ \\
\hline Ultrafast DSC & $10^{-5}-10^{0}$ & $0.22-0.55$ & $10^{-1}-10^{6} / 10^{-1}-10^{6}$ & $\begin{array}{c}\text { Non-Arrhenius kinetics }{ }^{c)} \\
{[12,21-23]}\end{array}$ \\
\hline Optical Excitations & $<10^{3}$ & $0.22-0.32$ & $10^{10}-10^{11} /$ up to $10^{12}$ & $\begin{array}{l}\text { Temperature simulations; } \\
\text { measures crystallized volume }\end{array}$ \\
\hline
\end{tabular}




\begin{tabular}{|c|c|c|c|c|}
\hline Electrical Measurements & $<10^{-1}$ & $0.22-0.32$ & $10^{0}-10^{8} /-$ & $\begin{array}{c}\text { fraction }^{\mathrm{d})}[24-26] \\
\text { Complex sample geometry }^{\mathrm{e})} \\
{[27,28]}\end{array}$ \\
\hline Direct Techniques & $\underset{\left(\mathrm{m} \mathrm{s}^{-1}\right)}{U_{\max }}$ & $T_{\mathrm{gu}}$ & $\begin{array}{l}\text { Heating/Cooling } \\
\text { Rate }\left(\mathrm{K} \mathrm{s}^{-1}\right)\end{array}$ & Limitations \\
\hline Levitation & $10^{-1}-10^{2}$ & $0.1-0.5$ & - & Dendritic growth $^{\mathrm{f})}[29]$ \\
\hline Dynamic TEM & $10^{-1}-10^{1}$ & $0.2-0.32$ & $10^{10}-10^{11} / 10^{10}$ & {$[30-32]$} \\
\hline Conventional TEM & $<10^{-5}$ & $0.4-0.7$ & $10^{-4}-10^{0} /-$ & $\begin{array}{l}\text { Sample preparation and e- } \\
\text { beam influence [33] }\end{array}$ \\
\hline Optical Microscopy ${ }^{\mathrm{g}}$ & $<10^{-2}$ & $0.4-0.7$ & - & - \\
\hline \multicolumn{5}{|c|}{$\begin{array}{l}\text { a) For fast crystal growth the crystal-liquid interface temperature does not correspond to the bulk temperature. } \\
\text { b) Because of the limited range of heating rates, crystallization occurs in a narrow temperature range in the supercooled liquid very close to } \\
T_{\mathrm{g}} \text {; the commonly used Kissinger analysis then gives the activation energy for crystal growth, } Q_{\mathrm{G}} \text {. } \\
\text { c) Because of extended heating rates, crystallization can occur over a wide temperature range in the supercooled liquid, revealing non- } \\
\text { Arrhenius kinetics. In this case, numerical modelling must be used to extract the temperature dependence of rates from the Kissinger data. } \\
\text { d) These techniques measure overall transformation rate, not } U(T) \text {. There is no direct measure of the temperature at the crystal-liquid } \\
\text { interface, which has to be estimated by modelling. } \\
\text { it } \\
\text { c) A thin-film sample is enclosed by electrodes and a dielectric. The mode and kinetics of crystallization can be strongly influenced by } \\
\text { stresses in the sample and by effects such as structural and chemical templating, and possible interdiffusion, at the interfaces between the } \\
\text { active layer and the electrodes. } \\
\text { f) The heat released during the crystallization gives a negative temperature gradient ahead of the crystal-liquid interface, typically leading to } \\
\text { instability of the solidification front and to dendritic growth. } \\
\text { g) Without using fast cameras. }\end{array}$} \\
\hline
\end{tabular}

Computer simulations are a special case; they are certainly useful (for example for pure metals) when experimental measurements are not available. In molecular-dynamics (MD) simulations, crystal growth rates in highly-unrelaxed (rejuvenated) glasses, not supercooled liquids, can be computed because of the high cooling rates (Fig. 1). The crystal growth rates that are of most interest in the present work have mostly been characterized by indirect techniques. These typically involve measurement of the overall rate of crystallization. That rate depends on the number of growth centres, or the crystal nucleation rate, in addition to the crystal growth rate; nevertheless approximations can be made to estimate values of $U$. Calorimetric measurements have so far been based on heating at a constant rate. At a higher rate, the crystallization mainly occurs at a higher temperature. But numerical simulations of the heat evolution show that the maximum in $U$ cannot be directly detected; instead samples fail to crystallize completely before $T_{\mathrm{m}}$ is reached on heating [23].

\section{Mechanisms of fast growth}

The value of $T_{\max } / T_{\mathrm{m}}$ is a result of the interplay between kinetic (dominant at low temperature) and thermodynamic (dominant at high temperature) factors. Around a homologous temperature of 0.55 , it is the kinetic factor that leads to the wide separation of the $U$ values for a pure metal $(\mathrm{Ag})$ and for a metallic-glass-forming system $\left(\mathrm{Cu}_{50} \mathrm{Zr}_{50}\right)$, as seen in Fig. 2. As shown by the intervening data for $\mathrm{Ge}_{2} \mathrm{Sb}_{2} \mathrm{Te}_{5}$, this regime of potentially fast growth is likely to be of interest for device applications, such as phase-change memory (PCM). For such an application, the active materials must have a GFA that is low, in order to ensure fast crystal growth; at the same time the glassy phase once formed must be sufficiently 
stable to retain the stored data. The wide range of growth behaviour between pure metals and systems such as $\mathrm{Cu}_{50} \mathrm{Zr}_{50}$ must provide opportunities to find materials with properties attractive for phase-change applications, possibly even improving on the chalcogenides that are typically used. This could be a new direction for metallic-glass research; for decades this has focused on finding systems of higher GFA, but it may now be useful to explore lower GFA.

Most treatments of crystal growth in this regime still focus on the classical models for diffusion-limited and collision-limited growth. In the diffusion-limited model, it is assumed that crystal growth relies on thermally activated diffusive-type jumps of the atoms at the crystal-liquid interface; in this case the limiting velocity (in the thermodynamic limit when all jumps are from liquid to crystal) is of the order of $10 \mathrm{~m} \mathrm{~s}^{-1}$. But experiments, particularly on supercooled pure metals, show that this velocity can be exceeded [34]. Furthermore, the phenomenon of solute trapping shows that the velocity of the crystal-liquid interface can exceed the diffusive speed of solute in the liquid [35].

To cope with this, it was suggested that, for single-component metallic liquids, there need be no thermal activation of atomic jumps at the crystal-liquid interface; rather the atoms shuffle into place. In this collision-limited growth [36], the rate is limited by the sound velocity in the liquid $U_{\text {kin }}^{\mathrm{c}} \propto(B / \rho)^{1 / 2}$, where $B$ is the adiabatic bulk modulus and $\rho$ is the density of the liquid. The kinetic factor $U_{\text {kin }}^{\text {c }}$ was found, by computer simulations [37], to be proportional to $U_{\mathrm{kin}}^{\mathrm{c}} \propto C \sqrt{\left(R / M T_{\mathrm{m}}\right)}$, for cubic close-packed (ccp) metals, where $M$ is the molar mass and $C$ is a constant varying with interfacial orientation: $C_{(100)}=1.3, C_{(110)}=1$ and $C_{(111)}$ ranges between $0.5-0.9$. There is thus a significant growth-rate anisotropy with $U_{\text {kin }}^{\mathrm{c}}(100)>U_{\text {kin }}^{\mathrm{c}}(110)>U_{\text {kin }}^{\mathrm{c}}(111) \quad[38,39]$. Also, for body-centred cubic (bcc) iron, $U_{\text {kin }}^{\text {c }}(100)>U_{\text {kin }}^{\text {c }}(110)$ [39]. According to such models, crystal growth rates in supercooled metals can be as high as $\sim 10^{3} \mathrm{~m} \mathrm{~s}^{-1}$, and rates as high as $\sim 10^{2} \mathrm{~m} \mathrm{~s}^{-1}$ have been observed experimentally. MD simulations suggest that such high growth rates are preserved far below $T_{\mathrm{g}}$. But the recent experimental observations by Zhong et al. [1] suggest that, at least for refractory metals below $T_{\mathrm{g}}$, the crystal growth rate must be much lower. The discrepancy between the computer simulations and the experimental observations emphasizes the need for better understanding of crystal growth in metallic systems.

There are problems: many measured growth rates are not fitted well by either model (diffusion-limited or collision-limited) and seem to lie in an intermediate regime [40]. Even the MD simulations of crystal growth in pure-metal systems [41] that (as noted above) show values of $U$ higher than observed experimentally, show a maximum in $U(T)$. Therefore to some extent the crystal growth must be thermally activated, contrary to the classical collisionlimited model.

Existing approaches focus on the transition, at increasing supercooling, from diffusionlimited growth permitting solute partitioning, to collision-limited growth enforcing solute 
trapping (i.e. partitionless solidification). But such a transition can account for no more than three orders of magnitude change in growth rate [35], and in any case does not treat different regimes within partitionless solidification. That such different regimes exist was shown by the work of Spaepen and Lin [42] on picosecond laser-induced quenching (cooling rate $\approx 10^{12}$ $\mathrm{K} \mathrm{s}^{-1}$ ) of alloys in the system $\mathrm{Fe}_{1-x} \mathrm{~B}_{x}$. Without any solute-trapping transition (the crystal growth is always partitionless), the growth rate can be suppressed from $10^{2} \mathrm{~m} \mathrm{~s}^{-1}$ for $x<3$ at. $\%$ to less than $10^{-1} \mathrm{~m} \mathrm{~s}^{-1}$ for higher B content, $x>5$ at.\%; in this way, at higher B content a glass is formed, even though, in the presence of a crystal/melt interface, there is no nucleation barrier. Glass formation in this system is controlled by $U(T)$ (Fig. 5). For B contents $x>10$ at.\% the GFA of the Fe-B system is good enough for the glassy state to be achievable by melt-spinning.

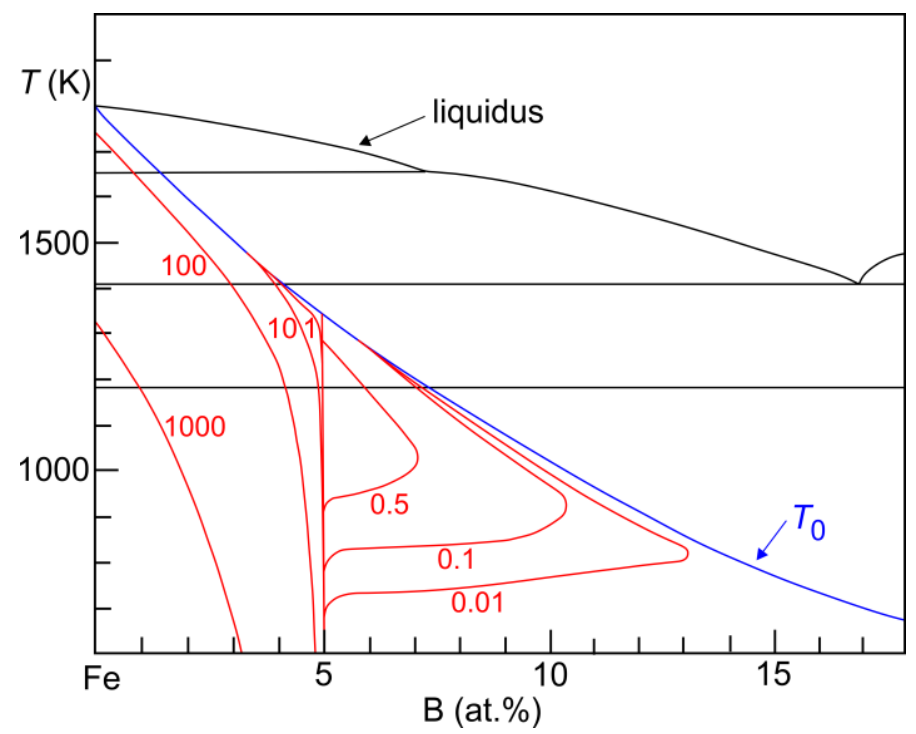

Fig. 5. Laser-induced melting and quenching of Fe-B alloys. The contour lines superposed on the phase diagram correspond to isokinetic crystal growth rates (labelled in $\mathrm{m} \mathrm{s}^{-1}$ ). $T_{0}$ is the equilibrium temperature for partitionless freezing to the bcc Fe(B) solid solution. Redrawn from Ref. [42].

Specifically, we conclude that the wide separation of the $U$ values for pure metals (e.g. $\mathrm{Ag})$ and for a metallic-glass-forming system $\left(\mathrm{Cu}_{50} \mathrm{Zr}_{50}\right.$, extrapolated according to the diffusion-limited model) seen at a homologous temperature of $\sim 0.55$ in Fig. 2 , is difficult to treat with current crystal-growth models.

Some guidance may be obtained from molecular systems. There are cases of polymorphic (i.e. congruent) crystal growth below $T_{\mathrm{g}}$; this glass-crystal growth has been measured, for example in ROY (Fig. 2), OTP, IMC and other organic (molecular) glasses $[43,44]$. These are of particular interest as the growth rate is not limited by bulk diffusion in the glass or the liquid, but by local conditions in a very thin $(\sim 3 \mathrm{~nm}$ thick) glass/crystal interface [43], or maybe by propagating fracture at the interface [45]. On supercooling, 
crystal growth follows the diffusion-limited model from $T_{\mathrm{m}}$ down to a characteristic temperature of 1.1-1.2T , where there is a breakdown in the Stokes-Einstein relation between viscous flow and diffusion. Below this temperature, $U$ increasingly deviates to values as much as $10^{4}$ times higher than predicted, and the value remains high even below $T_{\mathrm{g}}$. There are several models to account for this [46], yet none can fully explain the observed growth mode.

Whatever the details, this glass-crystal growth must be significantly decoupled from (i.e. is much faster than would be predicted from) the viscosity of the liquid. Crystal growth in pure-metal systems may represent an extreme case of such decoupling, and this may explain the instability of some as-deposited high-energy amorphous states [2].

\section{Fragile-to-strong crossover}

Many authors have pointed out that supercooled liquids such as water [47], metallicglass-forming systems [48,49] and yttrium oxide-aluminium oxide [50,51] can undergo a fragile-to-strong crossover on cooling. The existence of such crossover is not only of fundamental interest but, as recently shown for $\mathrm{Ag}_{5.5} \mathrm{In}_{6.5} \mathrm{Sb}_{59} \mathrm{Te}_{29}[52,53]$ and for $\mathrm{Te}_{85} \mathrm{Ge}_{15}$ [54], it may help to improve data retention in non-volatile chalcogenide-based phase-change memory (PCM). PCM exploits the contrast between high-resistance glassy and lowresistance crystalline phases, in archetypical materials such as $\mathrm{Ge}_{2} \mathrm{Sb}_{2} \mathrm{Te}_{5}$ and (Ag,In)-doped $\mathrm{Sb}_{2} \mathrm{Te}$ (AIST), to store information. Memory switching is achieved by reversible glass $\leftrightarrow$ crystalline transitions induced by electrical pulses: more intense pulses melt the crystal and the glass is formed on the subsequent rapid quenching; less intense pulses crystallize the glass. Contradictory requirements must be met: the memory must be stable against spontaneous crystallization; but fast recording, for which crystal growth is the ratelimiting step, must be ensured. A fragile-to-strong crossover on cooling the liquid (Fig. 6) may assist in meeting the requirements. The much-studied liquid $\mathrm{Ge}_{2} \mathrm{Sb}_{2} \mathrm{Te}_{5}$ shows a high fragility $(m=90)$ that provides a good description of the temperature dependence of its viscosity throughout the temperature range of interest. In contrast, AIST shows more complex behaviour. The high-temperature liquid is fragile $(m=74)$ facilitating fast switching (recording), while the low-temperature liquid is strong $(m=37)$ improving resistance against spontaneous crystallization at ambient temperature [53]. 


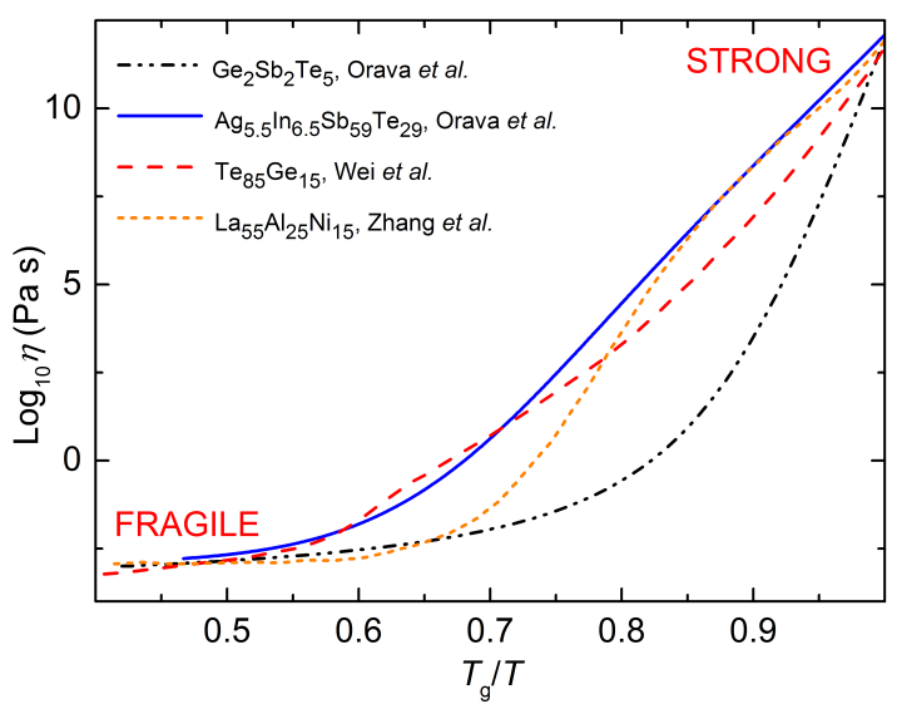

Fig. 6. Angell plot of viscosity showing the fragile-to-strong crossover on cooling liquid of phase-change materials $\mathrm{Ag}_{5.5} \mathrm{In}_{6.5} \mathrm{Sb}_{59} \mathrm{Te}_{29}$ [52,53], $\mathrm{Te}_{85} \mathrm{Ge}_{15}$ [54], and the metallic glass $\mathrm{La}_{55} \mathrm{Al}_{25} \mathrm{Ni}_{15}$ [56]. The phase-change chalcogenide $\mathrm{Ge}_{2} \mathrm{Sb}_{2} \mathrm{Te}_{5}$ [21] is an example of a highly fragile liquid.

For chalcogenide systems of interest for PCM, measurements of viscosity are possible around $T_{\mathrm{g}}$ and in the equilibrium liquid (above $T_{\mathrm{m}}$ ), but not over most of the intervening temperature range, in which the supercooled liquid crystallizes too readily. It is then useful that this no-man's land in viscosity data can be explored, albeit indirectly and partially, through measurements of crystal growth rates using the techniques considered in Fig. 3 and Table 1. In any case, in a variety of chalcogenides, the existence of a kinetic crossover now seems clear [52-55].

In-situ studies on oxides [50] and metallic glass-forming liquids [48] reveal structural changes in these liquids and suggest an existence of a first-order transition. It is puzzling that the crossover in phase-change chalcogenides [52-55] is much weaker than that found in metallic liquids [57]. Orava et al. [52] proposed that the existence of a crossover in AIST can be due to a change from metallic to semiconducting bonding on cooling, as was also suggested for pure tellurium [58]. Whether a crossover is sharp or not can be expressed by the ratio of the apparent fragilities $m^{\prime} / m$, where $m^{\prime}$ is the fragility of the high-temperature liquid. For AIST [53], and $\mathrm{Te}_{85} \mathrm{Ge}_{15}$ [54], $\mathrm{m}^{\prime} / m=2$, and the crossover is gradual and spread over a wide temperature range. Purely metallic liquids, such as those studied by Zhang et al. [56], have a sharper crossover $m^{\prime} / m=3-8$. The recent study by Stolpe et al. [48] shows a sharp increase in viscosity by 4 orders of magnitude in a rather narrow temperature range, $\Delta\left(T_{\mathrm{g}} / T\right)=0.1$, on cooling liquid $\mathrm{Zr}_{58.5} \mathrm{Cu}_{15.6} \mathrm{Ni}_{12.8} \mathrm{Al}_{10.3} \mathrm{Nb}_{2.8}$.

It is clear that possible fragile-to-strong crossovers need further study. They would also, of course, directly affect $U(T)$ and thereby the general correlations of interest in this review. It has already been noted that they may help to explain the differences in the crystallization behaviour of different chalcogenide phase-change systems (growth-dominated vs nucleation- 
dominated) [52,53]. Such behaviour also depends on crystal nucleation rates, which in AIST may be 6 orders of magnitude lower than in the highly-fragile liquid $\mathrm{Ge}_{2} \mathrm{Sb}_{2} \mathrm{Te}_{5}$, because of the crossover [52]. Similarly, Laksmono et al. [59] measured a 5 orders of magnitude decrease in nucleation rate in supercooled water due to the fragile-to-strong crossover.

Mode-coupling theory and other fundamental analyses of the dynamics of glass-forming liquids suggest a characteristic temperature, typically in the range (1.1-2.0) $T_{\mathrm{g}}$, at which there is a crossover in behaviour [60-63], variously considered in terms of the onset of nonergodicity or of dynamical cooperativity on cooling. The temperature of the maximum growth rate, $T_{\max } \approx 1.48 T_{\mathrm{g}}$, lies in the middle of these suggested crossovers. In the present work, we do not explore any link with the underlying dynamics, but treat the temperaturedependent liquid viscosity only descriptively in terms of fragility and fragile-to-strong crossover.

\section{Conclusions}

Marginal glass-forming systems that show fast crystal growth are of current interest. They show that better models are needed for crystal growth in liquids, even for such comparatively simple systems as pure metals. The ability for fast switching between glassy and crystalline states is applicable in phase-change memory, and better understanding of crystal growth kinetics may enable the development of intrinsically better materials for memory and related applications. We have reviewed that a range of methods is being developed for both direct and indirect measurement of the growth rate in systems with poor glass-forming ability. In this way, some data are now available, even for poor-glass-forming systems, for the growth rate over the entire temperature range from the glass transition to the melting point. Fitting the kinetics of crystal growth requires both the reduced glass-transition temperature and the kinetic fragility of the liquid to be taken into account. These parameters largely govern the viscosity of the liquid, but to derive the crystal growth rate there are additional factors to consider such as possible decoupling of interfacial rearrangements from viscous flow. Present studies do offer the prospect of better understanding of both glassforming ability and glass stability.

\section{Acknowledgements}

We acknowledge support from the World Premier International Research Center Initiative (WPI), MEXT, Japan.

\section{References}


[1] L. Zhong, J. Wang, H. Sheng, Z. Zhang, S.X. Mao, Formation of monatomic metallic glasses through ultrafast liquid quenching, Nature 512 (2014) 177-180.

[2] A.L. Greer, New horizons for glass formation and stability, Nat. Mater. 14 (2015) 542546.

[3] D.M. Herlach, D.M. Matson, Solidification of Containerless Undercooled Melts, Wiley-VCH, Weinheim, 2012.

[4] W. Wang, L.-M. Wang, M.Z. Ma, S. Binder, T. Volkmann, D.M. Herlach, J.S. Wang, Q.G. Xue, Y.J. Tian, R.P. Liu, Diffusion-controlled crystal growth in deeply undercooled melt on approaching the glass transition, Phys. Rev. B 83 (2011) 014202.

[5] A.L. Greer, Y. Sun, Stored energy in metallic glasses due to strains within the elastic limit, Philos. Mag. 96 (2016) 1643-1663.

[6] S.C. Glade, R. Busch, D.S. Lee, W.L. Johnson, R.K. Wunderlich, H.J. Fecht, Thermodynamics of $\mathrm{Cu}_{47} \mathrm{Ti}_{34} \mathrm{Zr}_{11} \mathrm{Ni}_{8}, \mathrm{Zr}_{52.5} \mathrm{Cu}_{17.9} \mathrm{Ni}_{14.6} \mathrm{Al}_{10} \mathrm{Ti}_{5}$ and $\mathrm{Zr}_{57} \mathrm{Cu}_{15.4} \mathrm{Ni}_{12.6} \mathrm{Al}_{10} \mathrm{Nb}_{5}$ bulk metallic glass forming alloys, J. Appl. Phys. 87 (2000) $7242-7248$.

[7] J. Orava, A.L. Greer, Fast and slow crystal growth kinetics in glass-forming melts, J. Chem. Phys. 140 (2014) 214504.

[8] V.A. Shneidman, D.R. Uhlmann, Crystallization kinetics in a glass-forming melt with retarded viscosity, J. Non-Cryst. Solids 223 (1998) 48-52.

[9] D. Turnbull, Under what conditions can a glass be formed? Contemp. Phys. 10 (1969) 473-488.

[10] C.A. Angell, Formation of glasses from liquids and biopolymers, Science 267 (1995) 1924-1935.

[11] O. Senkov, Correlation between fragility and glass-forming ability of metallic alloys, Phys. Rev. B 76 (2007) 104202.

[12] E. Zhuravlev, V. Madhavi, A. Lustiger, R. Androsch, C. Schick, Crystallization of polyethylene at large undercooling, ACS Macro Lett. 5 (2016) 365-370.

[13] M.D. Ediger, P. Harrowell, L. Yu, Crystal growth kinetics exhibit a fragility-dependent decoupling from viscosity J. Chem. Phys. 128 (2008) 034709.

[14] J.H. Na, M.D. Demetriou, M. Floyd, A. Hoff, G.R. Garrett, W.L. Johnson, Compositional landscape for glass formation in metal alloys, Proc. Natl. Acad. Sci. 111 (2014) 9031-9036.

[15] W.L. Johnson, J.H. Na, M.D. Demetriou, Quantifying the origin of metallic glass formation, Nat. Commun. 7 (2016) 10313.

[16] J.W.P. Schmelzer, A.S. Abyzov, V.M. Fokin, C. Schick, E.D. Zanotto, Crystallization of glass-forming liquids: Maxima of nucleation, growth, and overall crystallization rates, J. Non. Cryst. Solids 429 (2015) 24-32.

[17] M.I. Mendelev, M.J. Rahman, J.J. Hoyt, M. Asta, Molecular-dynamics study of solidliquid interface migration in fcc metals, Model. Simul. Mater. Sci. Eng. 18 (2010) 074002.

[18] W.-L. Chan, R.S. Averback, Y. Ashkenazy, Anisotropic atomic motion at undercooled crystal/melt interfaces, Phys. Rev. B 82 (2010) 020201. 
[19] D.W. Henderson, Thermal analysis of non-isothermal crystallization kinetics in glass forming liquids, J. Non. Cryst. Solids 30 (1979) 301-315.

[20] K.F. Kelton, Analysis of crystallization kinetics, Mater. Sci. Eng. A. 226-228 (1997) $142-150$.

[21] J. Orava, A.L. Greer, B. Gholipour, D.W. Hewak, C.E. Smith, Characterization of supercooled liquid $\mathrm{Ge}_{2} \mathrm{Sb}_{2} \mathrm{Te}_{5}$ and its crystallization by ultrafast-heating calorimetry, Nat. Mater. 11 (2012) 279-283.

[22] B. Chen, J. Momand, P.A. Vermeulen, B.J. Kooi, Crystallization kinetics of supercooled liquid Ge-Sb based on ultrafast calorimetry, Cryst. Growth Des. 16 (2016) $242-248$.

[23] J. Orava, A.L. Greer, Kissinger method applied to the crystallization of glass-forming liquids: Regimes revealed by ultra-fast-heating calorimetry, Thermochim. Acta 603 (2014) 63-68.

[24] M. Salinga, E. Carria, A. Kaldenbach, M. Bornhöfft, J. Benke, J. Mayer, et al., Measurement of crystal growth velocity in a melt-quenched phase-change material, Nat. Commun. 4 (2013) 2371.

[25] P. Zalden, A. von Hoegen, P. Landreman, M. Wuttig, A.M. Lindenberg, How supercooled liquid phase-change materials crystallize: Snapshots after femtosecond optical excitation, Chem. Mater. 27 (2015) 5641-5646.

[26] A.L. Greer, Diffusion and reaction in thin films, Appl. Surf. Sci. 86 (1995) 329-337.

[27] R.G.D. Jeyasingh, S.W. Fong, J. Lee, Z. Li, K.-W. Chang, D. Mantegazza, et al., Ultrafast characterization of phase-change material crystallization properties in the meltquenched amorphous phase, Nano Lett. 14 (2014) 3419-3426.

[28] A. Sebastian, M. Le Gallo, D. Krebs, Crystal growth within a phase change memory cell, Nat. Commun. 5 (2014) 4314.

[29] D.M. Herlach, Containerless undercooling of drops and droplets, in: D.M. Herlach, D.M. Matson (Eds.), Solidification of Containerless Undercooled Melts, Wiley-VCH, Weinheim, 2012, pp. 1-30.

[30] M.R. Armstrong, K. Boyden, N.D. Browning, G.H. Campbell, J.D. Colvin, W.J. DeHope, et al., Practical considerations for high spatial and temporal resolution dynamic transmission electron microscopy, Ultramicroscopy 107 (2007) 356-367.

[31] M.K. Santala, B.W. Reed, T. Topuria, S. Raoux, S. Meister, Y. Cui, et al., Nanosecond in situ transmission electron microscope studies of the reversible $\mathrm{Ge}_{2} \mathrm{Sb}_{2} \mathrm{Te}_{5}$ crystalline $\Leftrightarrow$ amorphous phase transformation, J. Appl. Phys. 111 (2012) 024309.

[32] M.K. Santala, S. Raoux, G.H. Campbell, Kinetics of liquid-mediated crystallization of amorphous Ge from multi-frame dynamic transmission electron microscopy, Appl. Phys. Lett. 107 (2015) 252106.

[33] R. Pandian, B.J. Kooi, J.T.M. De Hosson, A. Pauza, Influence of electron beam exposure on crystallization of phase-change materials, J. Appl. Phys. 101 (2007) $53526-53529$.

[34] T. Volkmann, Measurements of crystal growth velocities in undercooled melts of metals, in: D.M. Herlach, D.M. Matson (Eds.), Solidification of Containerless Undercooled Melts, Wiley-VCH, Weinheim, 2012, pp. 239-259. 
[35] M.J. Aziz, W.J. Boettinger, On the transition from short-range diffusion-limited to collision-limited growth in alloy solidification, Acta Metall. Mater. 42 (1994) 527-537.

[36] S.R. Coriell, D. Turnbull, Relative roles of heat transport and interface rearrangement rates in the rapid growth of crystals in undercooled melts, Acta Metall. 30 (1982) 21352139.

[37] M. Amini, B. Laird, Kinetic coefficient for hard-sphere crystal growth from the melt, Phys. Rev. Lett. 97 (2006) 216102.

[38] D. Sun, M. Asta, J. Hoyt, Kinetic coefficient of Ni solid-liquid interfaces from molecular-dynamics simulations, Phys. Rev. B 69 (2004) 024108.

[39] Y. Watanabe, Y. Shibuta, T. Suzuki, A molecular dynamics study of thermodynamic and kinetic properties of solid-liquid interface for bcc iron, ISIJ Int. 50 (2010) 11581164.

[40] D.M. Herlach, P. Galenko, D. Holland-Moritz, Metastable Solids from Undercooled Melts, Elsevier, Amsterdam, 2007, p. 199.

[41] Y. Ashkenazy, R.S. Averback, Kinetic stages in the crystallization of deeply undercooled body-centered-cubic and face-centered-cubic metals, Acta Mater. 58 (2010) 524-530.

[42] F. Spaepen, C.J. Lin, Partitionless crystallization and glass formation in Fe-B alloys during picosecond pulsed laser quenching, in: Amorphous Metals and Non-Equilibrium Processing, Les Editions de Physique, Les Ulis, 1984, pp. 65-72.

[43] D. Musumeci, C.T. Powell, M.D. Ediger, L. Yu, Termination of solid-state crystal growth in molecular glasses by fluidity, J. Phys. Chem. Lett. 5 (2014) 1705-1710.

[44] Y. Sun, L. Zhu, K. L. Kearns, M. D. Ediger, L. Yu, Glasses crystallize rapidly at free surfaces by growing crystals upward, Proc. Natl. Acad. Sci. 108 (2011) 5990-5995.

[45] C.T. Powell, H. Xi, Y. Sun, E. Gunn, Y. Chen, M.D. Ediger, et al., Fast crystal growth in o-terphenyl glasses: A possible role for fracture and surface mobility, J. Phys. Chem. B 119 (2015) 10124-10130.

[46] Y. Sun, H. Xi, S. Chen, M.D. Ediger, L. Yu, Crystallization near glass transition: Transition from diffusion-controlled to diffusionless crystal growth studied with seven polymorphs, J. Phys. Chem. B 112 (2008) 5594-5601.

[47] C.A. Angell, Water II is a "strong" liquid, J. Phys. Chem. 97 (1993) 6339-6341.

[48] M. Stolpe, I. Jonas, S. Wei, Z. Evenson, W. Hembree, F. Yang, et al., Structural changes during a liquid-liquid transition in the deeply undercooled $\mathrm{Zr}_{58.5} \mathrm{Cu}_{15.6} \mathrm{Ni}_{12.8} \mathrm{Al}_{10.3} \mathrm{Nb}_{2.8}$ bulk metallic glass forming melt, Phys. Rev. B 93 (2016) 014201.

[49] S. Wei, F. Yang, J. Bednarcik, I. Kaban, O. Shuleshova, A. Meyer, et al., Liquid-liquid transition in a strong bulk metallic glass-forming liquid, Nat. Commun. 4 (2013) 2083.

[50] G.N. Greaves, M.C. Wilding, S. Fearn, D. Langstaff, F. Kargl, S. Cox, et al., Detection of first-order liquid/liquid phase transitions in yttrium oxide-aluminum oxide melts, Science 322 (2008) 566-570.

[51] P.F. McMillan, G.N. Greaves, M. Wilson, M.C. Wilding, D. Daisenberger, Polyamorphism and liquid-liquid phase transitions in amorphous silicon and supercooled $\mathrm{Al}_{2} \mathrm{O}_{3}-\mathrm{Y}_{2} \mathrm{O}_{3}$ liquids, in: H.E. Stanley (Ed.), Liquid Polymorphism,Wiley, 
Hoboken, 2013, pp. 309-353.

[52] J. Orava, D.W. Hewak, A.L. Greer, Fragile-to-strong crossover in supercooled liquid Ag-In-Sb-Te studied by ultrafast calorimetry, Adv. Funct. Mater. 25 (2015) 4851-4858.

[53] J. Orava, H. Weber, I. Kaban, A.L. Greer, Viscosity of liquid Ag-In-Sb-Te: Evidence of a fragile-to-strong crossover, J. Chem. Phys. 144 (2016) 194503.

[54] S. Wei, P. Lucas, C.A. Angell, Phase change alloy viscosities down to $T_{\mathrm{g}}$ using AdamGibbs-equation fittings to excess entropy data: A fragile-to-strong transition, J. Appl. Phys. 118 (2015) 034903.

[55] S. Stølen, T. Grande, H.-B. Johnsen, Fragility transition in $\mathrm{GeSe}_{2}-$ Se liquids, Phys. Chem. Chem. Phys. 4 (2002) 3396-3399.

[56] C. Zhang, L. Hu, Y. Yue, J.C. Mauro, Fragile-to-strong transition in metallic glassforming liquids, J. Chem. Phys. 133 (2010) 014508.

[57] C. Zhou, L. Hu, Q. Sun, H. Zheng, C. Zhang, Y. Yue, Structural evolution during fragile-to-strong transition in $\mathrm{CuZr}(\mathrm{Al})$ glass-forming liquids, J. Chem. Phys. 142 (2015) 064508.

[58] H.L. Luo, Metastable Amorphous Phase of Tellurium-Base Alloy, Ph.D. thesis, California Institute of Technology, 1964.

[59] H. Laksmono, T.A. McQueen, J.A. Sellberg, N.D. Loh, C. Huang, D. Schlesinger, et al., Anomalous behavior of the homogeneous ice nucleation rate in "no-man's land", J. Phys. Chem. Lett. 6 (2015) 2826-2832.

[60] S.M. Bhattacharyya, B. Bagchi, P.G. Wolynes, Facilitation, complexity growth, mode coupling, and activated dynamics in supercooled liquids, PNAS 105 (2008) 1607716082.

[61] F. Mallamace, C. Branca, C. Corsaro, N. Leone, J Spooren, S.-H. Chen, H.E. Stanley, Transport properties of glass-forming liquids suggest that dynamic crossover temperature is as important as the glass transition temperature, PNAS 107 (2010) 22457-22462.

[62] B. Schmidtke, N. Petzold, R. Kahlau, M. Hofmann, E.A. Rössler, From boiling point to glass transition temperature: Transport coefficients in molecular liquids follow threeparameter scaling, Phys. Rev. E 86 (2012) 041507.

[63] M.E. Blodgett, T. Egami, Z. Nussinov, K.F. Kelton, Proposal for universality in the viscosity of metallic liquids, Sci. Rep. 5 (2015) 13837. 